\title{
Contrast Extravasation on CT Angiography Predicts Hematoma Expansion and Mortality in Acute Traumatic Subdural Hemorrhage
}

\author{
J.M. Romero, H.R. Kelly, J.E. Delgado Almandoz, J. Hernandez-Siman, J.C. Passanese, M.H. Lev, and R.G. González
}

\begin{abstract}
BACKGROUND AND PURPOSE: The presence of active contrast extravasation at CTA predicts hematoma expansion and in-hospital mortality in patients with nontraumatic intracerebral hemorrhage. This study aims to determine the frequency and predictive value of the contrast extravasation in patients with aSDH.
\end{abstract}

MATERIALS AND METHODS: We retrospectively reviewed 157 consecutive patients who presented to our emergency department over a 9-year period with aSDH and underwent CTA at admission and a follow-up NCCT within 48 hours. Two experienced readers, blinded to clinical data, reviewed the CTAs to assess for the presence of contrast extravasation. Medical records were reviewed for baseline clinical characteristics and in-hospital mortality. aSDH maximum width in the axial plane was measured on both baseline and follow-up NCCTs, with hematoma expansion defined as $>20 \%$ increase from baseline.

RESULTS: Active contrast extravasation was identified in 30 of 199 discrete aSDHs (15.1\%), with excellent interobserver agreement $(\kappa=$ $0.80 ; 95 \% \mathrm{Cl}, 0.7-0.9$ ). The presence of contrast extravasation indicated a significantly increased risk of hematoma expansion (odds ratio, 4.5; 95\% Cl, 2.0-10.1; $P=.0001$ ) and in-hospital mortality (odds ratio, $7.6 ; 95 \% \mathrm{Cl}, 2.6-22.3 ; P=0.0004$ ). In a multivariate analysis controlled for standard risk factors, the presence of contrast extravasation was an independent predictor of aSDH expansion $(P=.001)$ and in-hospital mortality $(P=.0003)$.

CONCLUSIONS: Contrast extravasation stratifies patients with aSDH into those at high risk and those at low risk of hematoma expansion and in-hospital mortality. This distinction could affect patient treatment, clinical trial selection, and possible surgical intervention.

ABBREVIATIONS: aPTT = activated partial thromboplastin time; $\mathrm{aSDH}=$ acute traumatic subdural hemorrhage; $\mathrm{Cl}=$ confidence interval; $\mathrm{GCS}=\mathrm{Glasgow}$ Coma Scale; INR = international normalized ratio; $\mathrm{TBI}=$ traumatic brain injury

A cute traumatic subdural hemorrhage carries a mortality rate of $68 \%$ in patients who are in a coma at the time of presentation. ${ }^{1-4}$ The incidence of aSDH is approximately $21 \%$ in patients with severe $\mathrm{TBI}^{4}$ and decreases to $11 \%$ in patients with mild and moderate TBI. ${ }^{5}$ Mortality secondary to aSDH has been related to initial hematoma size, the presence of additional brain

Received August 22, 2012; accepted after revision October 20

From the Division of Neuroradiology (J.M.R., H.R.K., J.H.-S., M.H.L., R.G.G.), Department of Radiology, Massachusetts General Hospital, Harvard Medical School, Boston, Massachusetts; and Interventional Neuroradiology (J.E.D.A.), Abbott Northwestern Hospital Minneapolis, Minnesota.

This work was funded in part by the Doris Duke Charitable Foundation Clinical Research Program Scholarship.

Previously presented in part in poster format at: American Heart Association/American Stroke Association: International Stroke Conference 2010, February 2010, San Antonio, Texas.

Please address correspondence to Hillary R. Kelly, MD, Division of Neuroradiology, Department of Radiology, Massachusetts General Hospital, Gray 2, Room 273A, 55 Fruit St, Boston, MA 02114; e-mail: hkelly2@partners.org

http://dx.doi.org/10.3174/ajnr.A3434 injury, midline shift, comatose state, and delay in hematoma evacuation $>2$ hours after arrival to the emergency department. ${ }^{6,7}$ The decision to undertake surgical intervention versus expectant management of aSDH is based on hematoma size, the presence of midline shift, admission GCS score, and hematoma growth. ${ }^{8}$ Early hematoma evacuation $(<4$ hours) has been shown to improve intracranial pressure and therefore brain perfusion, with a decrease in mortality compared with delayed surgical intervention in comatose patients with severe TBI. ${ }^{4}$ Although a significant proportion of patients are treated nonoperatively (noncomatose patients and comatose patients with aSDH $<10 \mathrm{~mm}$ in width and/or $<5 \mathrm{~mm}$ of midline shift), a subset of these aSDHs will expand, necessitating delayed operative intervention. The strong relationship between mass effect and mortality suggests that hematoma expansion is probably deleterious for brain perfusion and clinical outcome. ${ }^{9}$ However, to date, no reliable predictors of aSDHs expansion in the initial 48 hours have been identified. Identifying such a predictor may be helpful in the clinical decision to triage patients to early surgical intervention versus expectant management. 
Prior studies have found that the presence of active contrast extravasation at CTA, defined as the spot sign, is a powerful predictor of hematoma expansion and in-hospital mortality in patients with primary intracerebral hemorrhage. ${ }^{9-15}$ However, to date, no studies have assessed the frequency and predictive value of this important finding in patients with aSDH.

In our emergency department, CTA of the head and neck is frequently performed in patients who present with craniocervical trauma to detect vascular injury. ${ }^{15,16}$ Subsets of these patients also have an associated aSDH. This study aims to determine the frequency and predictive value for hematoma expansion and inhospital mortality of the CTA contrast extravasation in patients with aSDH.

\section{MATERIALS AND METHODS Patient Selection}

A waiver of informed consent was obtained from our institutional review board, and, in compliance with the Health Insurance Portability and Accountability Act, we conducted a retrospective review of all adult patients who presented to our emergency department with aSDH and were evaluated with a CTA of the head over a period of 9 years. Our inclusion criteria were 1): patient age $\geq 18$ years, 2) the presence of aSDH on an NCCT, 3) evaluation with a CTA of the head and neck on admission, and 4) follow-up NCCT within 48 hours of the baseline CTA. Patients who underwent operative intervention or died before the follow-up NCCT were excluded but were evaluated for the presence of a contrast extravasation on the initial CTA in a separate subgroup analysis. Patients who were discharged from the hospital without a follow-up NCCT were also excluded from the primary analysis but were evaluated for the presence of contrast extravasation as a separate subgroup. Patient exclusion criteria were the presence of 1) an $\mathrm{aSDH} \leq 1 \mathrm{~mm}$ in maximum axial width or 2 ) incomplete coverage of the aSDH on either the initial or follow-up NCCT.

The decision to perform CTA evaluation in our patient cohort was based on the emergency department protocol defined by the modified Denver screening criteria for blunt cerebrovascular injury. ${ }^{16}$ According to these criteria, a CTA was obtained in patients with any neurologic abnormality not explained by the diagnosed injury, blunt trauma patients presenting with epistaxis, or patients with any 1 of the following risk factors: GCS score $\leq 6$, petrous bone fracture, diffuse axonal injury, cervical spine fracture, fracture through the foramen transversarium, and/or Lefort II or III facial fractures.

\section{Image Acquisition}

NCCTs of the head as well as CTA examinations were performed on 16- or 64-section CT scanners (LightSpeed, GE Healthcare, Milwaukee, Wisconsin). The NCCTs were performed by use of the axial technique with $120-140 \mathrm{kVp}, 170 \mathrm{~mA}$, and 5-mm section thickness reconstructions. CTA was performed by scanning from the vertex to aortic arch by use of the following parameters: pitch, 0.5; collimation, $1.25 \mathrm{~mm}$; maximal $\mathrm{mA}, 350 ; \mathrm{kVp}, 120$; field of view, $22 \mathrm{~cm}$. Nonionic contrast material $(80-100 \mathrm{~mL})$ was administered by power injector at $4-5 \mathrm{~mL} / \mathrm{s}$ into an antecubital vein with either a fixed 25-second delay between the onset of contrast injection and the start of scanning, or Smart-Prep (GE
Healthcare), a semi-automatic contrast bolus triggering technique. The resulting 1.25-mm-thick axial source images were digitally archived. Standard maximum intensity projection images of the major cervical arterial structures were created by the 3D laboratory at our institution. The effective radiation dose of the CT angiogram of the head is an additional $2-3 \mathrm{mSv}$.

\section{Image Analysis}

Two experienced neuroradiologists (J.M.R., 12 years of experience, and H.R.K, 5 years of experience), blinded to clinical data and patient outcome, retrospectively reviewed the NCCTs to determine, by consensus reading, the 1) aSDH location (categorized as frontal, temporal, parietal, occipital, tentorial and/or parafalcine), 2) aSDH side, 3) midline shift ( $\mathrm{mm}$ ), and 4) maximum aSDH width on any axial source image, independent from the location of the contrast extravasation $(\mathrm{mm})$. After at least a 2-week interval, the 1.25 -mm axial CTA source images were independently reviewed in "Spot Windows" (width, 200; level, 110) by the same neuroradiologists to determine the presence of active contrast extravasation according to the following radiologic criteria (previously defined as the spot sign in studies of primary intracerebral hemorrhage):

1) One or more focus of contrast pooling within the aSDH

2) Attenuation $\geq 120 \mathrm{HU}$

3) Discontinuous from any vasculature adjacent to the aSDH, particularly cortical veins

4) Of any size and morphology

Differences in reader interpretation for the presence of contrast extravasation were adjudicated by consensus.

At least 2 weeks after interpretation of the CTAs, the maximum aSDH width on any axial source image in the follow-up NCCTs was performed independently and blinded to the CTA categorization by the same 2 neuroradiologists. Acute traumatic subdural hemorrhage expansion was calculated as a percent change between the maximum aSDH axial width on the initial and follow-up NCCT. Significant aSDH expansion was defined as an increase in maximum aSDH axial width $\geq 20 \%$ from the initial NCCT. The threshold of $20 \%$ was used to avoid an error of measurement on the NCCT obtained with $2.5-\mathrm{mm}$ thickness, which is $\pm 12.5 \% .^{17}$

\section{Medical Record Review}

Medical records were reviewed for patient age, sex, mechanism of injury, history of hypertension, history of antiplatelet therapy, admission platelet count, admission coagulation status (INR and aPTT), admission systolic and diastolic blood pressure, admission GCS score, time interval between the initial and follow-up NCCT, and in-hospital mortality. Altered coagulation was defined as an admission INR $>1.2$ or aPTT $>36$ seconds.

\section{Statistical Analysis}

Statistical analysis was performed with the use of the SAS 9.1 software package (SAS Institute, Cary, North Carolina). Interobserver agreement for the identification of contrast extravasation was determined with the $\kappa$ statistic. Sensitivity, specificity, positive predictive value, negative predictive value, and likelihood ratios were calculated. Univariate analyses and multivariable lin- 
ear regression were used to assess the dependence of hematoma expansion and in-hospital mortality on the following baseline clinical and radiologic factors: patient age, sex, admission GCS score, admission aPTT and INR, initial maximum aSDH axial width, midline shift, and the presence of contrast extravasation.

Demographic dichotomous variables were compared with the use of a Fisher exact test for significance. The Mann-WhitneyWilcoxon test was used for continuous and ordinal variables. A $P$ value of $<.05$ was considered statistically significant.

\section{RESULTS}

Between January 1, 2000, and January 31, 2009, 870 adult patients with acute head and neck trauma were evaluated with CTA to assess for vascular injury according to our emergency department protocol, based on the modified Denver screening criteria as listed in the Materials and Methods section. Of these, 430 patients also had associated subdural hemorrhage identified in the original neuroradiology report; 127 of the 430 were excluded on review because of the presence of aSDH of $\leq 1 \mathrm{~mm}$ in maximum axial width or imaging features of a chronic subdural hemorrhage. The remaining 303 patients were determined to have aSDH on review. Of these, several could not be included in the study for various reasons: 15 patients died in the hospital without follow-up imaging; 47 patients went to the operating room for intervention before undergoing an additional NCCT; 20 patients went to the operating room before receiving their CTA and therefore could not be evaluated for contrast extravasation; 6 patients did not undergo CTA until several days after admission; 24 patients underwent NCCT $>48$ hours after the initial CTA; 31 patients were discharged from the hospital without any additional imaging; and 3 patients only had CTA imaging of the neck, which did not adequately cover the aSDH. The remaining 157 patients underwent both CTA and a follow-up NCCT within 48 hours of the initial CTA.

Hence, 157 patients with 199 discrete aSDHs met our study's inclusion criteria. Mean patient age was 52 years (median, 51 years; range, $18-94$ years); 117 patients were male (74.5\%), and 40 were female $(25.5 \%)$. The most frequent mechanism of injury was fall from standing and the most common location of the aSDH was the frontal convexity. The mean time interval between the initial and follow-up NCCT was 11.76 hours (standard deviation, 7.98 hours; median, 8.7 hours; range, 1.7-35.2 hours). Table 1 summarizes the baseline clinical and radiologic characteristics of the patient population.

Contrast extravasation was found in 27 of the 157 patients with aSDH (17.2\%). Three patients had $>1$ aSDH with contrast extravasation. We identified at least 1 focus of contrast extravasation in 30 of the 199 discrete aSDHs (15.1\%), with excellent interobserver agreement $(\kappa=0.80 ; 95 \%$ CI, 0.7-0.9). All discrepancies regarding the presence of contrast extravasation concerned a miss by 1 of the 2 readers. No vascular mimics were noted in the discrepancy group on the basis of the CTA interpretation. Table 2 depicts the baseline clinical and radiologic characteristics in patients with and without contrast extravasation.
Table 1: Baseline clinical and radiologic characteristics

\begin{tabular}{lc}
\hline \multicolumn{1}{c}{ Characteristic } \\
\hline Mean age, years (SD) & $52(22.5)$ \\
Sex & \\
Male (\%) & $117(75)$ \\
Female (\%) & $40(25)$ \\
Mean admission blood pressure, mm Hg & \\
Systolic (SD) & $140.7(26.8)$ \\
Diastolic (SD) & $76.4(14.8)$ \\
Mean admission GCS (SD) & $11.58(4.17)$ \\
History of hypertension (\%) & $46(29)$ \\
Mean admission platelet count (SD) & $244(71)$ \\
Mean admission INR (SD) & $1.24(0.53)$ \\
Mean admission aPTT (SD) & $25.70(5.33)$ \\
Altered coagulation (\%) & $9(6)$ \\
Antiplatelet therapy (\%) & $24(15)$ \\
Mechanism of injury & \\
Fall from standing (\%) & $84(54)$ \\
Fall from height (\%) & $18(12)$ \\
Motor vehicle crash (\%) & $24(15)$ \\
Pedestrian struck (\%) & $16(10)$ \\
Direct blunt trauma (\%) & $13(8)$ \\
Penetrating trauma (\%) & $2(1)$ \\
aSDH location & \\
Frontal (\%) & $99(50)$ \\
Temporal (\%) & $38(19)$ \\
Parietal (\%) & $31(16)$ \\
Parafalcine (\%) & $15(8)$ \\
Occipital (\%) & $13(6)$ \\
Cerebellar (\%) & $3(1)$ \\
Mean initial maximum aSDH width, mm (SD) & $6.0(3.5)$ \\
Mean initial maximum midline shift, mm (SD) & $2.2(2.9)$ \\
Mean time to follow-up NCCT, hours (SD) & $11.76(7.98)$ \\
\hline & \\
&
\end{tabular}

\section{Accuracy of Contrast Extravasation for the Prediction of Expansion in aSDH}

Forty-six of the 199 aSDHs demonstrated significant hematoma expansion on follow-up NCCT (23.1\%). Fifteen of the 30 contrast extravasation-positive aSDHs demonstrated significant hematoma expansion (50\%), whereas only 31 of the 169 contrast extravasation negative-aSDHs expanded $(18.3 \%, P=.0025)$ (Fig 1). In univariate analysis, the presence of contrast extravasation significantly increased the risk of hematoma expansion in our patient population (positive predictive value, $50.0 \%$; odds ratio, $4.5 ; 95 \%$ CI, $2.0-10.1 ; P=.0004)$. The mean percentage volume change for the contrast extravasation-positive aSDH group was $25 \%$, whereas for the contrast extravasation-negative aSDH group, the mean change was $-3.6 \%(P<.0001)$. Importantly, among aSDHs that demonstrated hematoma expansion, those with contrast extravasation demonstrated a significantly larger mean percentage expansion (54.5\%) compared with those without contrast extravasation $(31.7 \%, P=.002)$. When the presence of contrast extravasation was entered into the multivariate logistic regression model as a binary variable (in addition to admission GCS score, maximum initial axial aSDH width, midline shift, and admission INR and aPTT), the presence of contrast extravasation became an independent predictor of expansion $(P=.0001)$. Table 3 summarizes the accuracy of the contrast extravasation for the prediction of hematoma expansion and in-hospital mortality in our patient population. 
Table 2: Comparison of baseline characteristics between patients with and without spot sign

\begin{tabular}{lccc}
\multicolumn{1}{c}{ Characteristic } & $\begin{array}{c}\text { Spot Sign } \\
(\boldsymbol{n}=\mathbf{2 7})\end{array}$ & $\begin{array}{c}\text { No Spot Sign } \\
(\boldsymbol{n}=130)\end{array}$ & $\begin{array}{c}\boldsymbol{P} \\
\text { Value }\end{array}$ \\
\hline $\begin{array}{l}\text { Mean age, years (SD) } \\
\text { Sex (\%) }\end{array}$ & $59(22.2)$ & $50(22.4)$ & $.07^{\mathrm{a}}$ \\
$\quad$ Male & $23(85)$ & $94(72)$ & $.22^{\mathrm{b}+}$ \\
$\quad$ Female & $4(15)$ & $36(28)$ & \\
Admission blood pressure, mm Hg & & & \\
$\quad$ Systolic (SD) & $144.4(30.5)$ & $140.0(26.1)$ & $.50^{\mathrm{a}}$ \\
$\quad$ Diastolic (SD) & $76.7(15.9)$ & $76.4(14.7)$ & $.93^{\mathrm{a}}$ \\
Mean admission GCS (SD) & $10.2(4.3)$ & $11.8(4.1)$ & $.09^{\mathrm{c}}$ \\
History of hypertension (\%) & $13(48)$ & $33(25)$ & $.02^{\mathrm{b}}$ \\
Mean admission platelet count (SD) & $235.4(85.5)$ & $246.2(68.2)$ & $.47^{\mathrm{a}}$ \\
Mean admission INR (SD) & $1.3(0.8)$ & $1.2(0.5)$ & $.27^{\mathrm{a}}$ \\
Mean admission aPTT (SD) & $27.4(6.3)$ & $25.4(5.1)$ & $.08^{\mathrm{a}}$ \\
Altered coagulation (\%) & $2(7)$ & $7(5)$ & $.48^{\mathrm{b}}$ \\
Antiplatelet therapy (\%) & $6(22)$ & $18(14)$ & $.38^{\mathrm{b}}$ \\
Mean initial maximum aSDH width, mm (SD) & $6.9(3.6)$ & $5.8(3.5)$ & $.12^{\mathrm{a}}$ \\
Mean initial maximum midline shift, mm (SD) & $0.40(0.4)$ & $0.18(0.3)$ & $.0004^{\mathrm{a}}$ \\
Mean time to follow-up NCCT, hours (SD) & $9.58(7)$ & $11.28(7.3)$ & $.29^{\mathrm{a}}$ \\
\hline
\end{tabular}

a Student $t$ test.

${ }^{\mathrm{b}}$ Fisher exact test.

cMann-Whitney test.

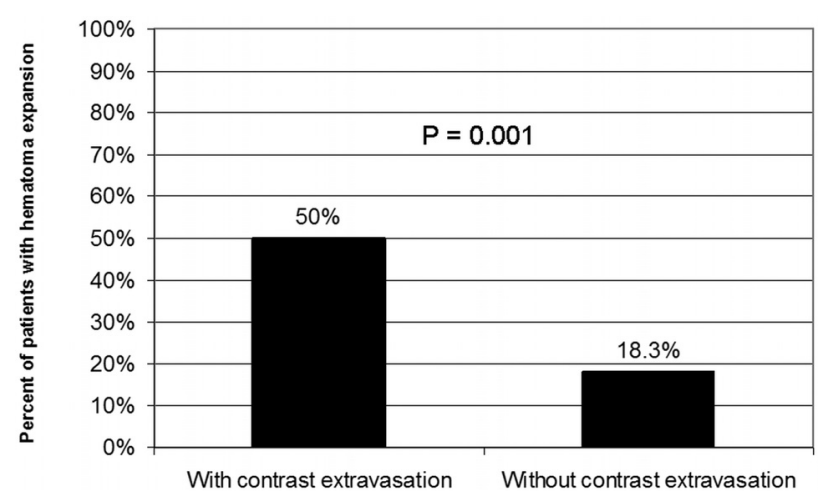

FIG 1. Relationship between the presence of contrast extravasation and the percentage of aSDH that demonstrated expansion shows a larger proportion of expanding aSDH in the group with contrast extravasation.

Table 3: Accuracy of the contrast extravasation (spot sign) for the prediction of hematoma expansion and in-hospital mortality in patients with aSDH

\begin{tabular}{lcc}
\hline & $\begin{array}{c}\text { Hematoma } \\
\text { Expansion }^{\text {a }}(95 \% \mathrm{Cl})\end{array}$ & $\begin{array}{c}\text { In-Hospital } \\
\text { Mortality (95\% CI) }\end{array}$ \\
\hline Sensitivity & $32.6(20.0-48.1)$ & $52.9(28.5-76.1)$ \\
Specificity & $90.2(84.1-94.2)$ & $87.1(80.2-92.0)$ \\
PPV & $50.0(31.7-68.3)$ & $33.3(17.2-54.0)$ \\
NPV & $81.7(74.8-87.0)$ & $93.8(87.8-97.1)$ \\
Positive LR & $3.3(1.8-6.3)$ & $4.1(2.2-7.6)$ \\
Negative LR & $0.75(0.61-0.91)$ & $0.54(0.33-0.90)$ \\
Accuracy & 76.9 & 83.4 \\
Prevalence & 23.1 & 10.8 \\
\hline
\end{tabular}

Note:- LR indicates likelihood ratio; NPV, negative predictive value; PPV, positive predictive value.

${ }^{\text {a }}$ Defined as an increase $\geq 20 \%$ from the initial maximum axial aSDH width.

\section{Accuracy of Contrast Extravasation for the Prediction of Mortality in aSDH}

Seventeen patients in our cohort died during hospitalization $(10.8 \%)$. Nine of the 27 patients with contrast extravasation died (33.3\%), whereas only 8 of the 130 patients without contrast ex- travasation died $(6.2 \%)$. The time between presentation and death ranged from 9 hours to 26.5 days, with a mean time to death of 8 days. In univariate analysis, the presence of contrast extravasation significantly increased the risk of inhospital mortality in our patient population (positive predictive value, 33.3\%; odds ratio, 7.6; 95\% CI, 2.6-22.3; $P=.0004$ ) (Fig 2). In univariate analysis, the significant risk factors for in-hospital mortality were the presence of contrast extravasation, admission GCS score, initial midline shift, admission INR, and admission aPTT (Table 4). In multivariate analysis controlling for admission GCS score, maximum initial axial aSDH width, midline shift, and admission INR and aPTT, contrast extravasation was an independent predictor of in-hospital mortality (odds ratio, 8.5; 95\% CI, 2.7-27.3; $P=.0003)$.

\section{Analysis of Patients Without Follow-Up Imaging}

We separately analyzed hematoma expansion and mortality in 3 distinct groups of patients who were not included in the primary analysis for expansion or mortality because of the lack of follow-up imaging. These groups included patients who died in the hospital, those who went to the operating room before follow-up imaging, and those who were discharged from the hospital without follow-up imaging.

There were 57 discrete aSDHs in the 47 patients who went to the operating room before undergoing follow-up NCCT. We identified at least 1 focus of contrast extravasation in 26 of the 57 discrete aSDHs (45.6\%). There were 32 discrete aSDHs identified in the 31 patients who were discharged from the hospital without follow-up imaging. Only one focus of contrast extravasation was identified in this cohort (3.1\%). Both of these groups were significantly different from the population at study, with a higher incidence of contrast extravasation in the early operative group $(P<$ $.0001)$ and a lower incidence of contrast extravasation in the early discharge group $(P<.0001)$.

Of the 15 patients who died in the hospital before undergoing follow-up NCCT, we detected 21 discrete aSDHs in the 15 patients (Fig 3). We identified at least 1 focus of contrast extravasation in 6 of the 21 discrete aSDHs (28.6\%). No significant difference was noted compared with the group with follow-up imaging $(P=.0578)$.

\section{DISCUSSION}

We have demonstrated that contrast extravasation is present in approximately $15 \%$ of patients with posttraumatic aSDH who undergo CTA evaluation in the emergency department, based on the modified Denver screening criteria to evaluate for vascular injury, and is an independent predictor of in-hospital mortality and expansion in this specific patient population. Furthermore, among patients with aSDH who had hematoma expansion, those 


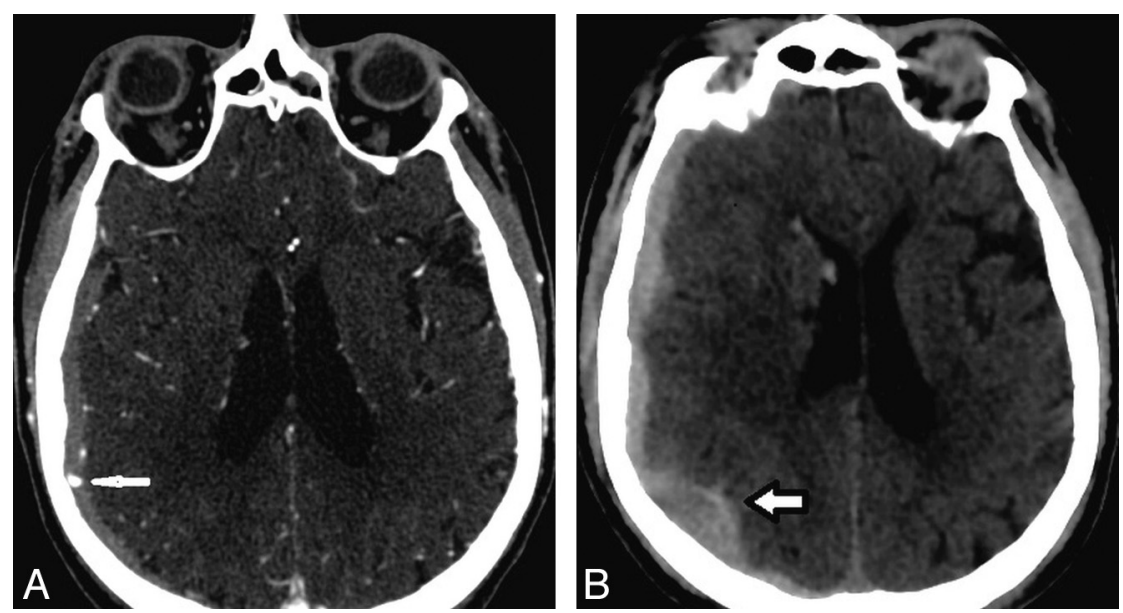

FIG 2. CT images of a 75-year-old man with blunt craniocervical trauma related to a motor vehicle crash. A, Axial source image from the CTA of the head in the emergency department demonstrates contrast extravasation (arrow) in the right hemispheric aSDH. B, Follow-up axial NCCT of the head at approximately the same level (obtained 4 hours later) demonstrates an increase of $>20 \%$ of the aSDH thickness (arrow).

Table 4: Comparison of clinical and radiologic characteristics between surviving and nonsurviving patients with aSDH

\begin{tabular}{lccc}
\multicolumn{1}{c}{ Characteristic } & $\begin{array}{c}\text { Survived } \\
(\boldsymbol{n}=140)\end{array}$ & $\begin{array}{c}\text { Died } \\
(\boldsymbol{n}=17)\end{array}$ & $\begin{array}{c}\boldsymbol{P} \\
\text { Value }\end{array}$ \\
\hline Mean age, years (SD) & $51(22.4)$ & $59(23.2)$ & $.16^{\mathrm{a}}$ \\
Sex (\%) & & & $.56^{\mathrm{b}}$ \\
$\quad$ Male & $103(74)$ & $14(82)$ & \\
$\quad$ Female & $37(26)$ & $3(18)$ & \\
Mean admission blood pressure, mm Hg & & & \\
$\quad$ Systolic (SD) & $139.9(26.5)$ & $147(29.5)$ & $.37^{\mathrm{a}}$ \\
$\quad$ Diastolic (SD) & $76.3(14.9)$ & $78.2(14.7)$ & $.66^{\mathrm{a}}$ \\
Mean admission GCS (SD) & $12(3.9)$ & $8(4.8)$ & $.0003^{\mathrm{c}}$ \\
History of hypertension (\%) & $38(27)$ & $8(47)$ & $.10^{\mathrm{b}}$ \\
Mean admission platelet count (SD) & $247.4(70.4)$ & $219.7(76.2)$ & $.13^{\mathrm{a}}$ \\
Mean admission INR (SD) & $1.2(0.45)$ & $1.6(1.0)$ & $.015^{\mathrm{a}}$ \\
Mean admission aPTT (SD) & $25.4(5.1)$ & $28.5(6.7)$ & $.022^{\mathrm{a}}$ \\
Altered coagulation (\%) & $7(5)$ & $2(12)$ & $.25^{\mathrm{b}}$ \\
Antiplatelet therapy (\%) & $19(14)$ & $5(30)$ & $.14^{\mathrm{b}}$ \\
Mean initial maximum aSDH width, mm (SD) & $6(3.6)$ & $6(3)$ & $.99^{\mathrm{a}}$ \\
Mean initial maximum midline shift, mm (SD) & $2(2.6)$ & $3.9(4.2)$ & $.011^{\mathrm{a}}$ \\
Patients with contrast extravasation (\%) & $18(13)$ & $9(53)$ & $.0004^{\mathrm{b}}$ \\
Patients without contrast extravasation (\%) & $122(87)$ & $8(47)$ & $.0004^{\mathrm{b}}$ \\
Mean time to follow-up NCCT, hours (SD) & $12(8.2)$ & $9.7(5.5)$ & $.27^{\mathrm{a}}$ \\
\hline
\end{tabular}

a Student $t$ test.

${ }^{b}$ Fisher exact test.

cMann-Whitney-Wilcoxon test.

who had contrast extravasation expanded to a larger degree than those without contrast extravasation. Given the excellent interobserver agreement for the identification of a focus of contrast extravasation in patients with aSDH, it is practical and feasible to use this CTA finding (within the mentioned diagnostic parameters) as a predictor of hematoma expansion in this clinical setting. Although not statistically significant, the higher rate of contrast extravasation in the cohort of excluded patients who died before undergoing follow-up imaging is also supportive of contrast extravasation as a predictor of poor outcome. The markedly high rate of contrast extravasation in the patients who underwent operative intervention soon after CTA suggests that there is a correlative relationship between this finding and clinical deterioration, though the presence or absence of hematoma expansion could not be assessed in these patients. Conversely, in the cohort of patients who were discharged from the hospital without follow-up imaging after the initial CTA, the presence of contrast extravasation was rare. Although this subgroup analysis was only performed in a small cohort of patients, this finding suggests that the absence of contrast extravasation could be reassuring and that patients with aSDH without contrast extravasation on imaging could be followed clinically without the need for multiple repeat imaging studies before discharge, thus preventing potentially unnecessary radiation exposure in this group.

According to the Centers for Disease Control and Prevention, from 2002 to 2006 there has been a $14.4 \%$ increase in the number of emergency department visits and a $19.5 \%$ increase in the number of hospitalizations for TBI. ${ }^{18}$ aSDH is a common feature of TBI, ${ }^{6,7}$ which results in serious clinical repercussions for survivors. Multiple radiologic findings have been associated with poor clinical outcome. A study evaluating NCCT findings at the "worst clinical time point" correlated hematoma thickness, midline shift, and status of the basal cisterns with poor outcome. ${ }^{19}$ Although our study did not demonstrate a significant difference in the initial maximum axial aSDH size between the survivors and those who died, we did find a significant difference in the degree of midline shift between these 2 patient groups. The discrepancy in the initial maximum axial aSDH size may be secondary to the smaller size of hematomas of our population (mean maximum axial size of $6 \mathrm{~mm}$ ) compared with those in prior reports $(11-16 \mathrm{~mm}){ }^{7}$

The in-hospital mortality rate in our patient cohort $(10.8 \%)$ is similar to the mortality rates reported in prior studies. ${ }^{18-21}$ The correlation between the presence of contrast extravasation and in-hospital mortality in patients with aSDH may be secondary to the presence of ongoing hemorrhage at the time of CTA evaluation, which results in hematoma expansion with increased axial aSDH size and midline shift as well as decreased regional cerebral blood flow-variables that have been related to decreased survival rates in prior reports. ${ }^{7,20}$ The cause of death in these patients is probably multifactorial and cannot be attributed to aSDH alone in most cases. These data are not able to support a specific clinical management strategy or intervention. However, the presence of contrast extravasation on CTA in similar patient populations could be used to identify patients at risk for in-hospital mortality regardless of their additional injuries and 

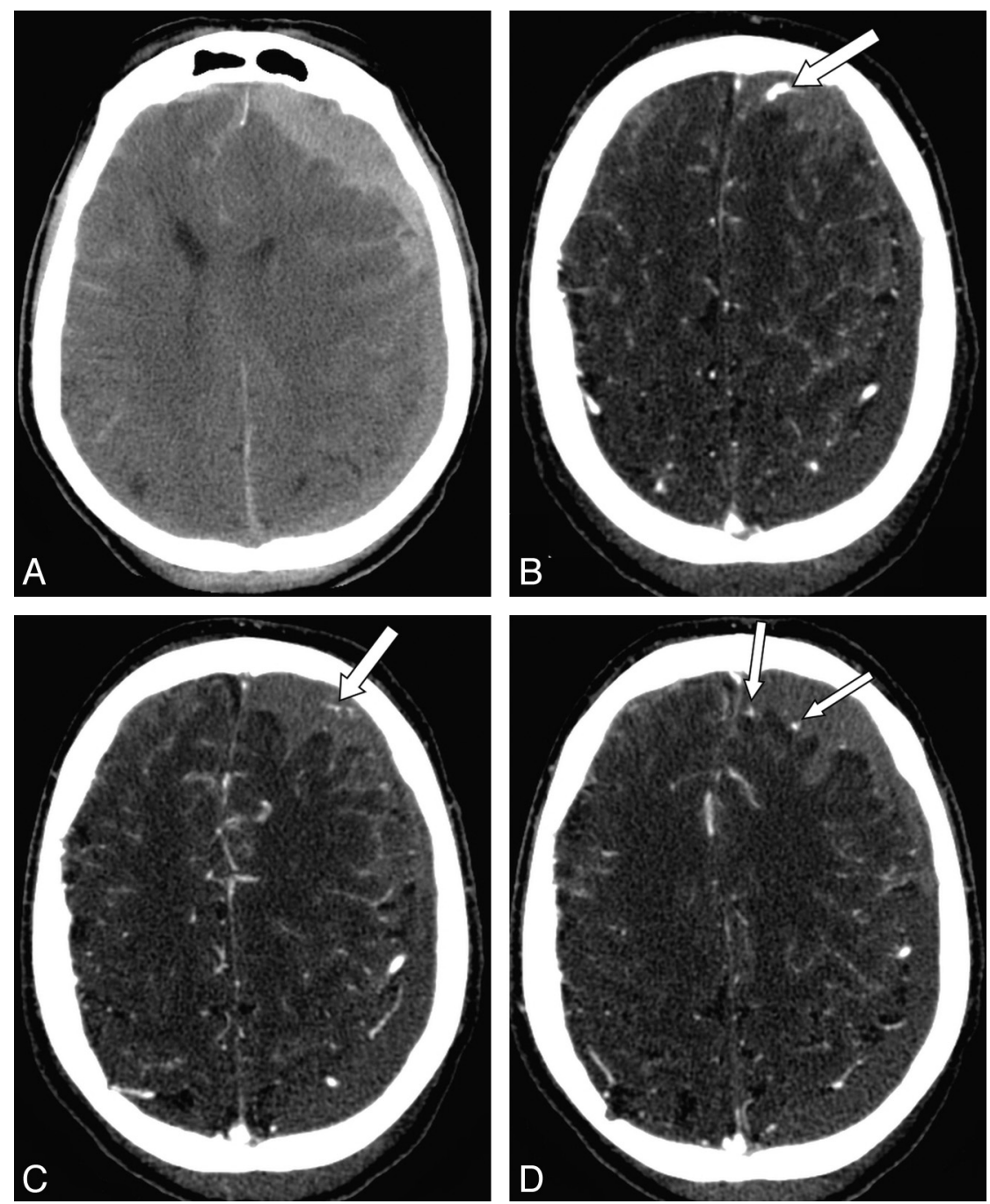

FIG 3. CT images of an 86-year-old man, status post fall. $A$, Axial image from the NCCT on admission demonstrates a left hemispheric aSDH and adjacent subarachnoid hemorrhage. $B, C$, and $D$, Images from the CTA acquired in the emergency department immediately after the NCCT demonstrate multiple foci of contrast extravasation (arrows) at various levels in the left frontal component of the aSDH. These foci of contrast extravasation were not contiguous with cortical vessels on review of the entire imaging study. The family declined further medical or surgical intervention, and the patient died in the emergency department shortly after the CTA was performed.

clinical status. Further prospective clinical studies would need to be performed to assess whether different clinical management strategies, such as early surgical intervention, would result in improved outcomes in this subset of patients with TBI.

One limitation of this study lies in the difficulty of assessing whether mortality was exclusively related to the aSDH. Other factors such as diffuse axonal injury, contusions, subarachnoid hemorrhage, and delayed surgical intervention ( $>4$ hours) have been shown to contribute to mortality and poor clinical outcome. ${ }^{4,22}$ This limitation is probably present in most of the aSDH and other TBI studies, and direct causality of death remains elusive in these cases. The selection of significant variables in the univariate analysis for the multivariate analysis was performed secondary to the limited number of observations and multiple number of variables. In addition, the smaller mean maximum axial aSDH size in this patient cohort compared with prior reports probably reflects a protocol of rapid surgical intervention in patients with larger $\mathrm{aSDH}$ at our institution. However, as a result of our exclusion of patients who underwent surgical evacuation before follow-up
NCCT, our cohort may represent a new subgroup of patients who currently would be treated nonoperatively but remain at risk for hematoma expansion and increased mortality. These results are limited to patients with similar characteristics based on the modified Denver screening criteria and should not be applied to all patients with aSDH. A minimally invasive CTA examination for the detection of contrast extravasation in aSDH could identify patients at increased risk for hematoma expansion and mortality, which could influence management and follow-up strategies in this subset of the TBI population.

\section{CONCLUSIONS}

The presence of contrast extravasation is highly specific for patients at risk of aSDH expansion and in-hospital mortality. aSDHs without contrast extravasation demonstrate minimal risk of expansion. This distinction could affect patient treatment, future clinical trial selection, and possibly surgical intervention. 
Disclosures: Javier Romero-UNRELATED: Board Membership: Lundbeck, Comments: Advisory Board of the DIAS trial. Ischemic Stroke trial; Consultancy: Lundbeck, Comments: Advisory Board of the DIAS trial. Ischemic Stroke trial; Grants/ Grants Pending: NIH.* Josser Delgado Almandoz-UNRELATED: Travel/ Accommodations/Meeting Expenses Unrelated to Activities Listed: Covidien/eV3. Michael Lev—RELATED: Grant: NIH, ${ }^{*}$ NINDS, ${ }^{*}$ Comments: SPOTRIAS grant; UNRELATED: Grants/Grants Pending: GE Healthcare* (* Money paid to institution).

\section{REFERENCES}

1. Gennarelli TA, Spielman GM, Langfitt TW, et al. Influence of the type of intracranial lesion on outcome from severe head injury. J Neurosurg 1982;56:26-32

2. Kotwica Z, Brzezinski J. Acute subdural haematoma in adults: an analysis of outcome in comatose patients. Acta Neurochir (Wien) 1993;121:95-99

3. Lobato RD, Cordobes F, Rivas JJ, et al. Outcome from severe head injury related to the type of intracranial lesion: a computerized tomography study. J Neurosurg 1983;59:762-74

4. Seelig JM, Becker DP, Miller JD, et al. Traumatic acute subdural hematoma: major mortality reduction in comatose patients treated within four hours. N Engl J Med 1981;304:1511-18

5. Massaro F, Lanotte M, Faccani G, et al. One hundred and twentyseven cases of acute subdural haematoma operated on: correlation between CT scan findings and outcome. Acta Neurochir (Wien) 1996;138:185-91

6. Haselsberger K, Pucher R, Auer LM. Prognosis after acute subdural or epidural haemorrhage. Acta Neurochir (Wien) 1988;90:111-16

7. Zumkeller M, Behrmann R, Heissler HE, et al. Computed tomographic criteria and survival rate for patients with acute subdural hematoma. Neurosurgery 1996;39:708-12

8. Bullock MR, Chesnut R, Ghajar J, et al. Surgical management of acute subdural hematomas. Neurosurgery 2006;58:S16-S24

9. Delgado Almandoz JE, Yoo AJ, Stone MJ, et al. Systematic characterization of the computed tomography angiography spot sign in primary intracerebral hemorrhage identifies patients at highest risk for hematoma expansion: the spot sign score. Stroke 2009; 40:2994-3000

10. Wada R, Aviv RI, Fox AJ, et al. CT angiography "spot sign" predicts hematoma expansion in acute intracerebral hemorrhage. Stroke 2007;38:1257-62

11. Delgado Almandoz JE, Yoo AJ, Stone MJ, et al. The spot sign score in primary intracerebral hemorrhage identifies patients at highest risk of in-hospital mortality and poor outcome among survivors. Stroke 2010;41:54-60

12. Goldstein JN, Fazen LE, Snider R, et al. Contrast extravasation on CT angiography predicts hematoma expansion in intracerebral hemorrhage. Neurology 2007;68:889-94

13. Becker KJ, Baxter AB, Bybee HM, et al. Extravasation of radiographic contrast is an independent predictor of death in primary intracerebral hemorrhage. Stroke 1999;30:2025-32

14. Kim J, Smith A, Hemphill JC 3rd, et al. Contrast extravasation on CT predicts mortality in primary intracerebral hemorrhage. AJNR Am J Neuroradiol 2008;29:520-25

15. Park SY, Kong MH, Kim JH, et al. Role of 'spot sign' on CT angiography to predict hematoma expansion in spontaneous intracerebral hemorrhage. J Korean Neurosurg Soc 2010;48:399-405

16. Biffl WL, Moore EE, Offner PJ, et al. Optimizing screening for blunt cerebrovascular injuries. Am J Surg 1999;178:517-22

17. Prionas ND, Ray S, Boone JM. Volume assessment accuracy in computed tomography: a phantom study. J Appl Clin Med Phys 2010;11:3037

18. Coronado VG, Xu L, Basavaraju SV, et al. Surveillance for traumatic brain injury-related deaths-United States, 1997-2007. In: Centers for Disease Control and Prevention, MMWR Surveill Summ 2011 May 6;60:1-32

19. Servadei F, Nasi MT, Giuliani G, et al. CT prognostic factors in acute subdural haematomas: the value of the 'worst' CT scan. Br J Neurosurg 2000;14:110-16

20. Howard MA 3rd, Gross AS, Dacey RG Jr, et al. Acute subdural hematomas: an age-dependent clinical entity. J Neurosurg 1989;71:858-63

21. Gazzola S, Aviv RI, Gladstone DJ, et al. Vascular and nonvascular mimics of the CT angiography "spot sign" in patients with secondary intracerebral hemorrhage. Stroke 2008;39:1177-83

22. Wong GK, Tang BY, Yeung JH, et al. Traumatic intracerebral haemorrhage: is the CT pattern related to outcome? Br J Neurosurg 2009;23:601-05 